\title{
Percepções de clientes com HIV/AIDS sobre a cartilha para o autoexame ocular
}

\section{Perceptions of clients with HIV/AIDS about the use of an educational guide for eye self- examination}

\section{Percepciones de clientes con VIH/SIDA acerca del uso de cartilla para el autoexamen ocular}

\author{
Jennara Candido do Nascimento'; Ellen Lucy Vale de SouzaII; Paulo Cesar de Almeida'III \\ Lorita Marlena Freitag Pagliuca ${ }^{I V}$; Joselany Afio CaetanoV
}

\begin{abstract}
RESUMO: Objetivou-se analisar percepções de clientes com o Vírus da Imunodeficiência Humana (HIV) e com a Síndrome da Imunodeficiência Adquirida (AIDS) durante o uso de cartilha para o autoexame ocular. Estudo descritivo e exploratório, com abordagem qualitativa, desenvolvido em ambulatório de hospital de referência no atendimento de doenças infecciosas, em Fortaleza - CE, com 130 pessoas com HIV/AIDS, de maio a agosto de 2010. Os dados foram coletados mediante entrevista semiestruturada e submetidos à análise crítica com base na literatura selecionada. A cartilha obteve aceitação favorável e os sujeitos apontaram as ilustrações como facilitadoras para o autoexame ocular. Contudo, destacaram como dificuldade a existência de termos técnicos e apresentaram sugestões, sobretudo a inclusão de mais figuras e a redução dos enunciados. As sugestões referidas estão contribuindo para reformular a cartilha, com vistas a torná-la mais adequada ao uso cotidiano, diminuindo as barreiras e facilitando a execução dos passos.
\end{abstract}

Palavras-Chave: Tecnologia educacional; promoção da saúde; infecções por HIV; saúde ocular.

\begin{abstract}
This study aimed at analyzing the perceptions of clients with Human Immunodeficiency Virus (HIV) and with the Acquired Immunodeficiency Syndrome (AIDS) while using an educational guide for eye self-examination. Descriptive and exploratory study with qualitative approach, developed from May to August 2010, in outpatient care of referral hospital in infectious diseases in Fortaleza, CE, Brazil, with 130 people living with HIV/AIDS. Data was collected through semi-structured interviews, and analyzed critically on the basis of selected literature. The educational guide received favorable acceptance, with the illustrations regarded as facilitators for the conduction of eye self-examination. However, technical terms were found difficult and suggestions were made, especially for the inclusion of more images and reduction of statements. The suggestions are helping rephrase the guide, and make it more suitable for everyday use, reducing barriers, and facilitating the performance of steps. Keywords: Educational technology; health promotion; HIV infections; eye health.
\end{abstract}

RESUMEN: El objetivo fue analizar las percepciones de clientes con el Virus de la Inmunodeficiencia Adquirida (VIH) y com el Síndrome de Inmunodeficiencia Adquirida (SIDA) durante el uso de cartilla para autoexamen ocular. Estudio descriptivo, exploratorio, con enfoque cualitativo, desarrollado en hospital de referencia de atención ambulatoria en enfermedades infecciosas, en Fortaleza - CE - Brasil, con 130 personas que viven con el VIH/SIDA, de mayo a agosto de 2010. Los datos fueron recolectados a través de entrevistas semiestructuradas y sometidos al análisis crítico, según la literatura seleccionada. La cartilla obtuvo aceptación favorable y los sujetos indicaron las ilustraciones como facilitadores para la realización del autoexamen ocular. Sin embargo, destacaron como dificultad la presencia de términos técnicos y presentaron sugerencias, en especial la inclusión de más figuras y la reducción de declaraciones. Las sugerencias están ayudando a remodelar la cartilla, haciendo que sea más adecuada para uso diario, reduciéndose barreras para facilitar el logro de los pasos.

Palabras Clave: Tecnología educacional; promoción de la salud; infecciones por VIH; salud ocular.

\section{INTRODUÇÃo}

Entre as complicações decorrentes da infecção pelo HIV, merecem destaque aquelas que comprometem o sistema visualVI, as quais se manifestam de forma inesperada, podendo ocasionar a cegueira ${ }^{1}$. Cerca de 50 a $75 \%$ das pessoas que convivem com o HIV e/ou com a Síndrome da Imunodeficiência Adquirida (AIDS) desenvolvem alguma complicação nos olhos. A maioria é desencadeada por infecções oportunistas e neoplasias, como resultado direto da imunossupressão $0^{2,3}$.

IDoutora em Enfermagem. Professora Adjunta III do Curso de Graduação em Enfermagem do Centro Universitário Estácio do Ceará. Brasil. E-mail: jennaracandido@yahoo.com.br

IIEnfermeira da Unidade de Terapia Intensiva, Hospital Geral de Fortaleza. Ceará, Brasil. E-mail: ellenlucy88@hotmail.com

IIIDoutor em Saúde Pública pela Universidade de São Paulo. Professor Adjunto do Curso de Graduação em Nutrição e Enfermagem e do Programa de Pós-Graduação da Universidade Estadual do Ceará. Brasil. E-mail: pc49almeida@gmail.com

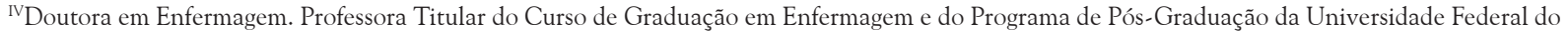
Ceará. Brasil. E-mail: pagliuca@ufc.br

voutora em Enfermagem. Professora Adjunta do Curso de Graduação em Enfermagem e do Programa de Pós-Graduação da Universidade Federal do Ceará. Brasil. E-mail: joselany@ufc.br

${ }^{\mathrm{V}}$ Artigo extraído da Dissertação Avaliação de uma tecnologia educativa na promoção da saúde ocular de pessoas portadoras do HIV/AIDS, apresentada em 2010 ao Programa de Pós-Graduação em Enfermagem, Universidade Federal do Ceará, Brasil. 
Diante de um quadro tão complexo, a possibilidade de perda visual parcial ou completa repercute negativamente na vida desses indivíduos, e, assim, compromete sua independência para o autocuidado e sua interação com o meio ao redor ${ }^{4}$. De acordo com estudos, a deficiência visual tem significado destrutivo, sendo muitas vezes percebida como um processo de finitude ${ }^{4-6}$.

Embora no contexto de cuidados, hospitalar e ambulatorial, essas pessoas contem com profissionais especializados, as práticas de saúde privilegiam abordagens relacionadas à adesão à terapia antirretroviral e ao manejo de comorbidades, a exemplo da tuberculose. Outras áreas de interesse, tal como a saúde ocular, encontram-se ainda pouco exploradas pela dinâmica de atendimento.

Desse modo, a pesquisa se justifica na medida em que o cuidado com os olhos, no contexto do HIV/ AIDS, é fator importante no processo saúde/doença, a partir da concepção de integralidade, ampliando o escopo de atuação dos profissionais de saúde para além de ações meramente curativas. Pelo exposto, objetivou-se analisar a percepção de pessoas que convivem com o HIV/AIDS sobre o uso da cartilha para o autoexame ocular.

\section{REVISÃo DE LITERATURA}

Ao analisar a produção científica relacionada à saúde ocular, foram identificadas experiências inerentes à prevenção e ao controle de agravos aos olhos no contexto escolar ${ }^{6-8}$. No entanto, ainda é grande a lacuna no tocante aos cuidados oculares entre as pessoas que convivem com o HIV/AIDS.

Com base em alguns estudos realizados, houve a necessidade de identificar, compreender e buscar estratégias eficazes para amenizar problemas oculares no âmbito do HIV/AIDS ${ }^{9,10}$. Para tal, construiu-se e validou-se cartilha para o autoexame ocular ${ }^{11}$. Esta foi formulada para ser utilizada pelas pessoas que convivem com HIV/AIDS em seu cotidiano como parte das ações de autocuidado. $O$ autoexame ocular consiste em uma série de técnicas simples as quais propiciam às pessoas avaliarem sua acuidade visual para perto/longe; as estruturas externas: pálpebras, cílios, conjuntivas, esclera, íris e pupila; movimento ocular; visão central/periférica. Essas etapas têm por objetivo identificar possíveis alterações, como: redução da acuidade visual.

Diante dos relatos de dificuldade dos usuários dos serviços de saúde para conseguir consulta com oftalmologista, a evidenciar fragilidade na atenção às pessoas soropositivas, além do alto custo dos exames oftalmológicos, constitui estratégia eficaz o autoexame ocular, que se caracteriza como prevenção secundária, com custo reduzido e seguro.

Outros estudos apresentam resultados do uso de cartilha para o ensino de usuários pelo enfermeiro ${ }^{12-19}$.
Nestes, é possível perceber a intenção de colaborar para o empoderamento dos sujeitos envolvidos, favorecendo o desenvolvimento de habilidades que os auxiliem a modificar comportamentos, vencer dificuldades e esclarecer dúvidas. Entretanto, apenas os estudos de Caetano e Pagliuca direcionaram-se para a construção de uma cartilha destinada à promoção da saúde ocular de pessoas soropositivas para o HIV $^{10}$.

\section{Metodologia}

Trata-se de estudo exploratório, descritivo, com abordagem qualitativa dos dados, em virtude da natureza do objeto do estudo e do objetivo proposto. A pesquisa foi realizada em hospital de referência no atendimento de doenças infecciosas, localizado em Fortaleza, Ceará, Brasil. Justifica-se a escolha por esse campo, pelo fato de a instituição ser referência no atendimento de doenças infecciosas, com especial destaque ao HIV/AIDS.

Foram selecionadas 130 pessoas que conviviam com o HIV/AIDS, todas em atendimento ambulatorial na referida instituição, nos meses de maio a agosto de 2010. Esse número foi definido pelo critério de saturação compreendido como o momento em que o acréscimo de dados e informações em uma pesquisa não altera a compreensão do fenômeno estudado ${ }^{20}$. Os participantes foram selecionados na sala de espera, durante as consultas médicas previamente agendadas. Como critérios de inclusão, foram considerados os seguintes parâmetros: ser alfabetizado, estar devidamente cadastrado no serviço anteriormente citado, ser maior de 18 anos e apresentar condições físicas para executar a técnica proposta para o autoexame ocular. Foram desconsiderados para fins de ingresso neste estudo os analfabetos, os que estavam aguardando atendimento no serviço para tratar outras doenças infecciosas, a exemplo das hepatites, e os cegos.

Para a coleta de dados, trabalhou-se de segunda a quinta-feira, de forma individual, mediante entrevista semiestruturada, após o uso da cartilha. Do formulário utilizado constavam duas partes: a primeira com dados de identificação (idade, sexo, renda, escolaridade, estado civil), dados relativos à infecção pelo HIV (tempo de diagnóstico e tratamento e tratamento farmacológico) e a segunda contendo uma questão norteadora, tendo como foco a percepção dos participantes quanto ao uso da cartilha para o autoexame ocular.

As percepções advindas desta questão foram organizadas e analisadas, por meio de abordagem qualitativa dos depoimentos, visando à classificação e categorização, e posteriormente submetidas à análise crítica, à luz da literatura selecionada, com base nas seguintes fases: transcrição dos relatos; codificação dos relatos segundo a proposta de análise; síntese e interpretação dos resultados ${ }^{21}$. 
Primeiramente, procedeu-se à leitura dos dados na íntegra, para apreensão inicial de seus conteúdos. Em continuidade, estes foram sendo estes agrupados por similaridade e frequência, sendo analisados em torno do eixo temático: facilidades e dificuldades na realização do autoexame ocular.

Em observância à legislação sobre a pesquisa em seres humanos ${ }^{22}$, o projeto de pesquisa foi submetido à apreciação do Comitê de Ética em Pesquisa da instituição, onde o estudo se desenvolveu, o qual foi aprovado conforme parecer $n^{\circ}$ 005/2010. Como parte da documentação, elaborou-se o Termo de Consentimento Livre e Esclarecido (TCLE), cujos participantes (P) foram informados sobre os objetivos da pesquisa, os procedimentos, riscos, desconfortos e benefícios. Para manter-lhes o anonimato, foram denominados como P1, P2, P3 e, assim, sucessivamente. Também se respeitou o desejo de participarem ou não.

\section{Resultados e Discussão}

\section{Perfil dos participantes}

A idade dos participantes variou entre 19 e 56 anos; $56(43,1 \%)$ estavam na faixa etária de 30 a 39 anos; e $40(30,8 \%)$ na faixa de 40 a 56 anos. Dos 130 entrevistados, $100(76,9 \%)$ eram do sexo masculino, $98(75,4 \%)$ eram solteiros e $76(58,5 \%)$ concluíram o ensino médio. Com relação à ocupação, a maioria, 95 $(73,1 \%)$, trabalhava em atividades formais e informais. Destas, as mais citadas foram as funções de professor - 9(7\%), comerciante - 8(6\%), cozinheiro - 5(4\%), cabeleireiro - $5(4 \%)$ e costureiro - 5(4\%). No referente à renda mensal, 59(45,4\%) ganhavam até um salário mínimo. Vale ressaltar que, em alguns casos, o valor informado foi ainda menor.

No tocante ao tempo de diagnóstico, 36(27,7\%) tinham entre 2 e 5 anos de confirmação diagnóstica, com média de $6 \pm 5$ anos. Esse também foi o tempo mais referido de tratamento, por $51(39,2 \%)$ dos participantes. Portanto, indicando que para este grupo o tempo de diagnóstico e de tratamento foi concomitante.

\section{Facilidades e dificuldades no autoexame ocular}

Ao longo dos discursos dos participantes, revelou-se a compreensão dos benefícios advindos do uso da cartilha para o autoexame ocular. Como se depreende, o material despertou neles o interesse pela temática da saúde ocular no contexto do HIV/ AIDS. Segundo observado, os relatos ressaltavam a importância dos desenhos para a realização dos passos, conforme exposto nos trechos a seguir:

Os desenhos são importantes para realização dos passos, pois eles mostram como eu devo fazer. (P10)

Se não tivessem os desenhos, eu não conseguiria fazer os passos. (P95)
Consoante evidenciado, os desenhos foram fundamentais para uma melhor compreensão dos exames, principalmente na avaliação da acuidade visual longe e das estruturas oculares externas. Denomina-se acuidade visual o grau em que detalhes e contornos dos objetos são notados; geralmente é definida em termos da distância mais curta pela qual duas linhas podem ser separadas e ainda percebidas como duas linhas ${ }^{23}$. Com o exame ocular externo podem ser detectados desvios oculares pela simples observação, opacificações da córnea ou alterações da sua forma e do seu diâmetro, entre outras ${ }^{24}$.

Um grande desafio no processo de ensino do autoexame ocular consiste no fato dele ser um método pouco conhecido quando comparado ao seu análogo autoexame das mamas. Portanto, menos utilizado no contexto de cuidados à população geral. Por sua vez, as dificuldades enfrentadas durante a execução do autoexame ocular estavam basicamente relacionadas à falta de compreensão de alguns termos existentes no texto, como por exemplo esclerótica. De acordo com os participantes, a linguagem deveria ser mais simples e se deveria evitar o uso de termos técnicos.

Acho que a linguagem deveria ser mais simples, mais popular. (P61)

Para a pessoa leiga, não é importante colocar os nomes: escala de Snellen, grade de Amsler [...]. Deveria substituir por nomes fáceis tipo: papel quadriculado. (P48)

Tive dificuldade para seguir alguns passos. (P126)

Um material escrito em linguagem facilmente entendível favorece a percepção e a satisfação do paciente, amplia o desenvolvimento das suas atitudes e habilidades, facilita-lhe a autonomia e a adesão às condutas de prevenção e tratamento ${ }^{25}$.

Também se considerou como incompreensão o fato de o indivíduo não ter reconhecido a importância de realizar o autoexame, mesmo na ausência de problemas oculares, conforme os relatos, a seguir:

Não consegui utilizá-la, porque não era do meu interesse, pois não utilizo óculos. (P44)

Não realizei os exames para perto e longe porque sabia que tinha miopia. (P11)

A cultura de procurar atendimento especializado, somente após a instalação dos problemas de ordem física ainda é bastante comum entre os indivíduos e coletivos, comprometendo o alcance das ações de saúde. Todavia, uma série de fatores afeta a busca pelo atendimento especializado, entre estes, o fator financeiro e o desconhecimento da população acerca da importância da avaliação frequente, mesmo na ausência de algum problema de saúde ${ }^{26}$.

Assim, a inclusão de tecnologias educativas nos serviços de saúde voltadas para a triagem visual de pessoas que convivem com o HIV/AIDS pode atuar na 
prevenção do surgimento de formas graves da doença ocular e incentivar o desenvolvimento de habilidades voltadas para o autocuidado. Cabe enfatizar: a anamnese e o exame oftalmológico realizados por pessoal capacitado, ainda que não especialistas, permitem diagnosticar e tratar determinadas condições oculares, referenciando ao oftalmologista quando necessário ${ }^{27}$.

Como proposto, o diálogo mediado por uma tecnologia educativa constitui-se uma forma de cuidado humanizado promotor da emancipação dos sujeitos $^{28,29}$. Desse modo, ênfase crescente sobre o conceito de gerenciamento de doenças crônicas tem sido observada em estudos, com a prestação de apoio clínico e educacional. Entretanto, para haver mudança de comportamento, se requer reforço contínuo durante determinado período de tempo ${ }^{30,31}$.

Portanto, a introdução da cartilha sobre autoexame ocular em consultas de enfermagem em ambulatórios torna-se reforço de ações de autocuidado realizadas por pessoas que convivem com o HIV/ AIDS. No entanto, esta técnica deve ser trabalhada de forma continuada. Assim, as pessoas poderão promover as avaliações com frequência e de forma adequada, buscando os serviços de saúde, quando houver alterações.

\section{CONClusão}

Nesta pesquisa foi possível identificar a percepção dos clientes com HIV/AIDS acerca da cartilha para o autoexame ocular. Evidenciou-se que apesar das dificuldades encontradas, nenhum participante deixou de efetuar o autoexame ocular com o uso da cartilha. Eles se empenharam em executar cada avaliação, com base nas orientações descritas e, ao final, manifestaram suas percepções sobre as facilidades e dificuldades vivenciadas.

Como limitação do estudo, destacou-se a não aplicação da cartilha para o autoexame ocular entre as pessoas analfabetas que convivem com o HIV/AIDS. Ainda, é necessário produzir outros trabalhos acerca dessa temática, envolvendo este grupo específico, uma vez que também apresenta risco para a ocorrência de comprometimento visual. Como esta tecnologia se propõe a promover a saúde das coletividades, é necessário ampliar seu uso para todo e qualquer indivíduo.

\section{REFERÊNCIAS}

1.Platnick J, Crum AV, Soohoo S, Cedeño PA, Johnson $\mathrm{MH}$. The globe: infection, inflammation, and systemic disease. Semin Ultrasound CT MRI. 2011; 32: 38-50. 2.Moayedi S. Head, neck and ophthalmologic manifestations of HIV in the emergency department. Emerg Med Clin North Am. 2010; 28: 265-71.
3.Gupta AK, Krishna V. Ocular manifesttation of AIDS. In: Gupta AK, Krishna V, editores. Clínical ophthalmology: contemporary perspectives. 9ª ed. India: Elsevier; 2009. p. 401-10.

4.Caetano JA, Pagliuca LMF. Autocuidado e o portador do HIV/AIDS: sistematização da assistência de enfermagem. Rev Latino-Am Enfermagem. 2006; 14: 51-61. 5.Vargas MA, Rodrigues MLV, Figueiredo JFC, Souza NV. Condições oftalmológicas de pacientes com síndrome da imunodeficiência adquirida com longo tempo de seguimento. Arq Bras Oftalmol. 2007; 70: 85-9.

6.Couto Júnior AS, Jardim JL, Oliveira DA, Gobetti TC, Portes AJF, Neurauter R. Alterações oculares em crianças pré-escolares e escolares no município de Duque de Caxias, Rio de Janeiro, Brasil. Rev Bras Oftalmol. 2010; 69: 7-11.

7.Lapa MCS, Freitas AM, Pedroso GC, Furusato MA, Ventura RN. Programa Embu Enxergando Melhor: uma proposta de atenção integral à saúde ocular em préescolares. Rev Paul Pediatr. 2008; 26: 113-8.

8.Granzoto JA, Ostermann CSPS, Brum LF, Pereira PG, Granzoto T. Avaliação da acuidade visual em escolares da $1^{\text {a }}$ série do ensino fundamental. Arq Bras Oftalmol. 2003; 66: 167-71.

9.Caetano JA, Pagliuca LM. Percepção visual alterada em pacientes com HIV/AIDS. Rev Baiana Enferm. 2002; 17: 65-74.

10.Caetano JA, Pagliuca LMF. Alterações oculares relacionadas com as manifestações clínicas da AIDS: uma avaliação para a intervenção de enfermagem. Rev enferm UERJ. 2000; 8: 101-7.

11.Zombini EV, Pelicioni MCF. Estratégias para avaliação de um material educativo em saúde ocular. Rev Bras Crescimento Desenvolv Hum. 2011; 21: 51-8.

12.Farias SMC, Teixeira OLC, Moreira W, Oliveira MAF, Pereira MO. Caracterização dos sintomas físicos de estresse na equipe de pronto atendimento. Rev esc enferm USP. 2010; 45: 722-9.

13.Fracolli LA, Chiesa AM. A percepção das famílias sobre a cartilha Toda hora é hora de cuidar. O Mundo da Saúde. 2010; 34: 36-42.

14.Martins J, Veríssimo MDLOR, Oliveira MA. Avaliação dos instrumentos do projeto Nossas Crianças: Janelas de Oportunidades, segundo agentes comunitários de saúde. Texto contexto-enferm. 2008; 17: 106-14.

15.Grippo MLVS, Fracolli LA. Avaliação de uma cartilha educativa de promoção ao cuidado da criança a partir da percepção da família sobre temas de saúde e cidadania. Rev esc enferm USP. 2008; 42: 430-6.

16.Fonseca LMM, Leite AM, Vasconcelos MGL, Castral TC, Scochi CGS. Cartilha educativa on line sobre os cuidados com o bebê pré-termo: aceitação dos usuários. Ciênc Cuid Saúde. 2007; 6: 238-44.

17.Chagas NR, Monteiro ARM. Educação em saúde e família: o cuidado ao paciente, vítima de acidente vascular cerebral. Acta Sci, Health Sci. 2006; 26: 193-204. 18. Fonseca LMM, Scochi CGS, Rocha SMM, Leite AM. Cartilha para orientação materna sobre os cuidados com o bebê prematuro. Rev Latino-Am Enfermagem. 2004; 12: 65-75. 
19.Caetano JA, Pagliuca LMF. Cartilha para o autoexame ocular para portadores do HIV/AIDS como tecnologia emancipatória: relato de experiência. Rev Eletr Enf [online]. 2006 [citado em 10 jan 2013]; 8: 241-9. Disponível em: www.fen.ufg.br/fen_revista/revista8_2/v8n2a09.htm. 20.Thiry-Cherques HR. Saturação em pesquisa qualitativa: estimativa empírica de dimensionamento. Rev PMKT. 2009; 3: 20-7.

21.Creswell JW. Projeto de pesquisa: método qualitativo, quantitativo e misto. $3^{\text {a }}$ ed. Porto Alegre (RS): Artmed; 2010.

22.Ministério da Saúde (Br). Conselho Nacional de Saúde. Resolução no196 de 10 de outubro de 1996. Aprova as diretrizes e normas regulamentadoras de pesquisa envolvendo seres humanos. Brasília(DF): Ministério da Saúde; 1996.

23. Barrett KE, Barman SM, Boitano S, Brooks HL. Fisiologia médica de Ganong. 24ํㅡㄹ ed. Porto Alegre (RS): McGraw-Hill; 2014.

24.Jarvis C. Guia de exame físico para a enfermagem. Rio de Janeiro: Elsevier; 2012.

25.Pommier J, Guevel MR, Jourdan D. Evaluation of health promotion in schools: a realistic evaluation approach using mixed methods. BMC Public Health. 2010;10: 43.
26.Castagno VD, Fassa AG, Silva MC, Carret MLV. Carência de atenção à saúde ocular no setor público: um estudo de base populacional. Cad Saúde Pública. 2009; 25: 2260-72.

27.Silva JV, Ferreira BFA, Pinto HS. Princípios da oftalmologia: avaliação oftalmológica. 2013 [citado em 12 out 2014] Disponível em: http://www.ligadeoftalmo.ufc.br/ arquivos/ed_principios__avaliacao_oftalmologica.pdf 28. Oliveria MS, Fernandes AFC, Sawada NO. Manual educativo para o autocuidado da mulher mastectomizada: um estudo de validação. Texto contexto-enferm. 2008; 17: 115-23.

29.Barros EJL, Santos SSC, Gomes GC, Erdmann AL. Gerontotecnologia educativa voltada ao idoso estomizado à luz da complexidade. Rev Gaúcha Enferm. 2012; 33: 95-101.

30.Song F, Holland R, Barton GR, Bachmann M, Blyth A, Maskrey V et al. Self-help materials for the prevention of smoking relapse: study protocol for a randomized controlled trial. Trials. 2012; 13:69.

31.Eckman MH, Wise R, Leonard AC, Dixon E, Burrows $\mathrm{C}$, Khan $\mathrm{F}$ et al. Impact of health literacy on outcomes and effectiveness of an educational intervention in patients with chronic diseases. Patient Educ Couns. 2012; 87 (2):143-51. 